\title{
Targeting p53 with SLMP53-2 for melanoma treatment: counteracting tumour proliferation, dissemination and therapeutic resistance
}

Joana Loureiro ${ }^{1}$, Liliana Raimundo ${ }^{2}$, Juliana Calheiros ${ }^{3}$, Valentina Barcherini ${ }^{4}$, Nuno Lima $^{5}$, Célia Gomes ${ }^{5}$, Inês Almeida ${ }^{6}$, Marco G. Alves ${ }^{7}$, José Costa $^{6}$, Maria MM Santos ${ }^{8}$, and Lucília Saraiva ${ }^{9}$

${ }^{1}$ Laboratório de Microbiologia, Departamento de Ciências Biológicas, Faculdade de Farmácia, Universidade do Porto, Rua de Jorge Viterbo Ferreira n. ํㅜ 164, 4050-313, Porto, Portugal

${ }^{2}$ Laboratório de Microbiologia, Departamento de Ciências Biológicas, Faculdade de

Farmácia, Universidade do Porto, Rua de Jorge Viterbo Ferreira n. ${ }^{0}$ 164, 4050-313

${ }^{3}$ Universidade do Porto Faculdade de Farmácia

${ }^{4} \mathrm{FFUL}$

${ }^{5}$ IBILI

${ }^{6}$ University of Porto Faculty of Medicine

${ }^{7}$ Universidade do Porto Instituto de Ciencias Biomedicas Abel Salazar

${ }^{8}$ Research Institute for Medicines (iMed.ULisboa), Faculty of Pharmacy, Universidade de

Lisboa, Av. Prof. Gama Pinto, 1649-003 Lisboa, Portugal

${ }^{9}$ University of Porto Faculty of Pharmacy

November 5, 2020

\begin{abstract}
Background and purpose: Melanoma is the deadliest form of skin cancer mostly due to its high metastatic propensity and therapeutic resistance in advanced stages. The frequent inactivation of the p53 tumour suppressor protein in melanomagenesis may predict promising outcomes for p53 activators in melanoma therapy. Herein, we aimed to investigate the anti-tumour potential of the p53-activating agent tryptophanol-derived oxazoloisoindolinone SLMP53-2 against melanoma. Experimental Approach: 2D/3D cell cultures and xenograft mouse models were used to unveil the anti-tumour activity and the underlying molecular mechanism of SLMP53-2 in melanoma. Key results: SLMP53-2 inhibited the growth of human melanoma cells in a p53-dependent manner through induction of cell cycle arrest and apoptosis. Notably, SLMP53-2 induced p53 stabilization by disruption of the p53-MDM2 interaction, with subsequent enhancement of p53 transcriptional activity. It also promoted the expression of p53-regulated microRNAs (miRNAs), including the tumour suppressors miR-145 and miR-23a. Moreover, it displayed anti-invasive and anti-migratory properties in melanoma cells, by inhibiting epithelial-to-mesenchymal transition (EMT), angiogenesis and extracellular lactate production. Importantly, SLMP53-2 did not induce resistance in melanoma cells. In addition, it synergised with vemurafenib, dacarbazine and cisplatin, and re-sensitized vemurafenib-resistant melanoma cells. SLMP53-2 also exhibited antitumor activity in human melanoma xenograft mouse models by repressing cell proliferation and EMT, while stimulating cell death. Conclusions and implications: This work discloses the p53-activating agent SLMP53-2 with promising therapeutic potential in advanced melanoma, either as a single agent or in combination therapy. By targeting p53, SLMP53-2 may counteract major features of melanoma aggressiveness, particularly metastasis and therapeutic resistance.
\end{abstract}




\section{Hosted file}

Manuscript_Loureiro et al.pdf available at https://authorea.com/users/373264/articles/491001targeting-p53-with-slmp53-2-for-melanoma-treatment-counteracting-tumour-proliferationdissemination-and-therapeutic-resistance

A

B

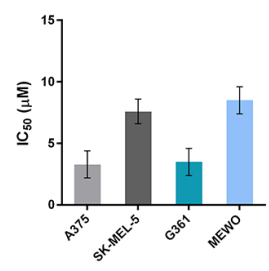

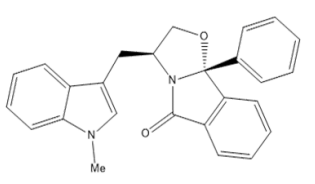

C

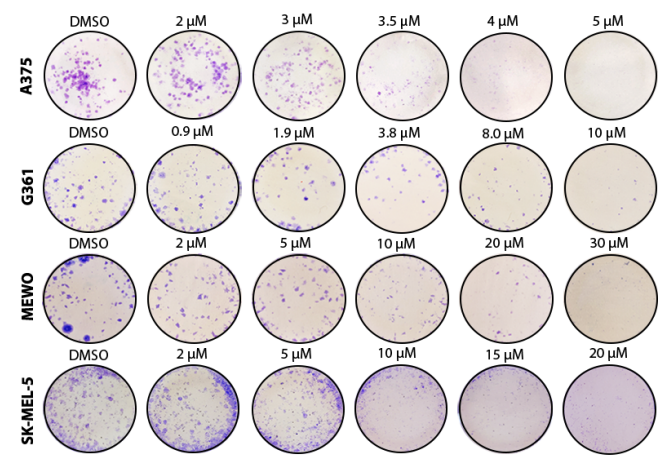

D

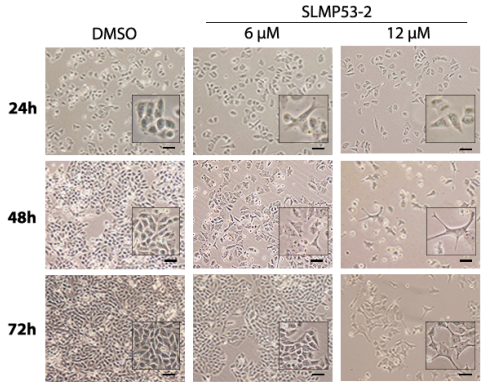

G

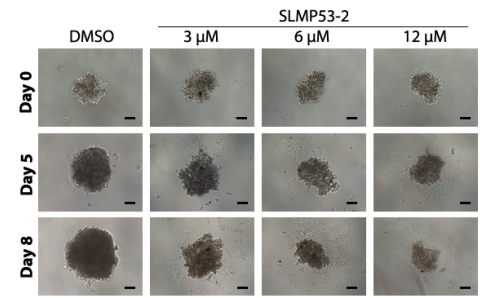

I

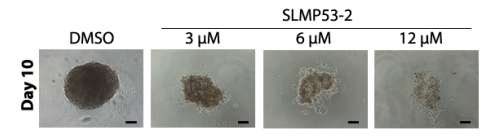

E

F

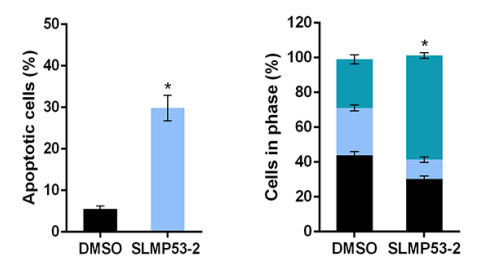

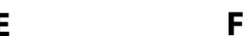

H
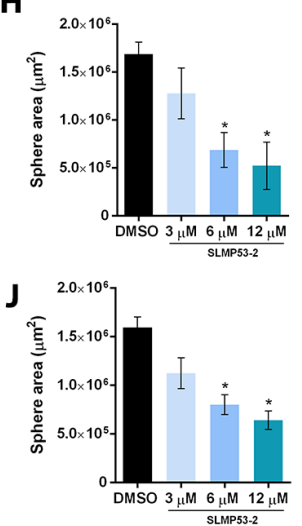

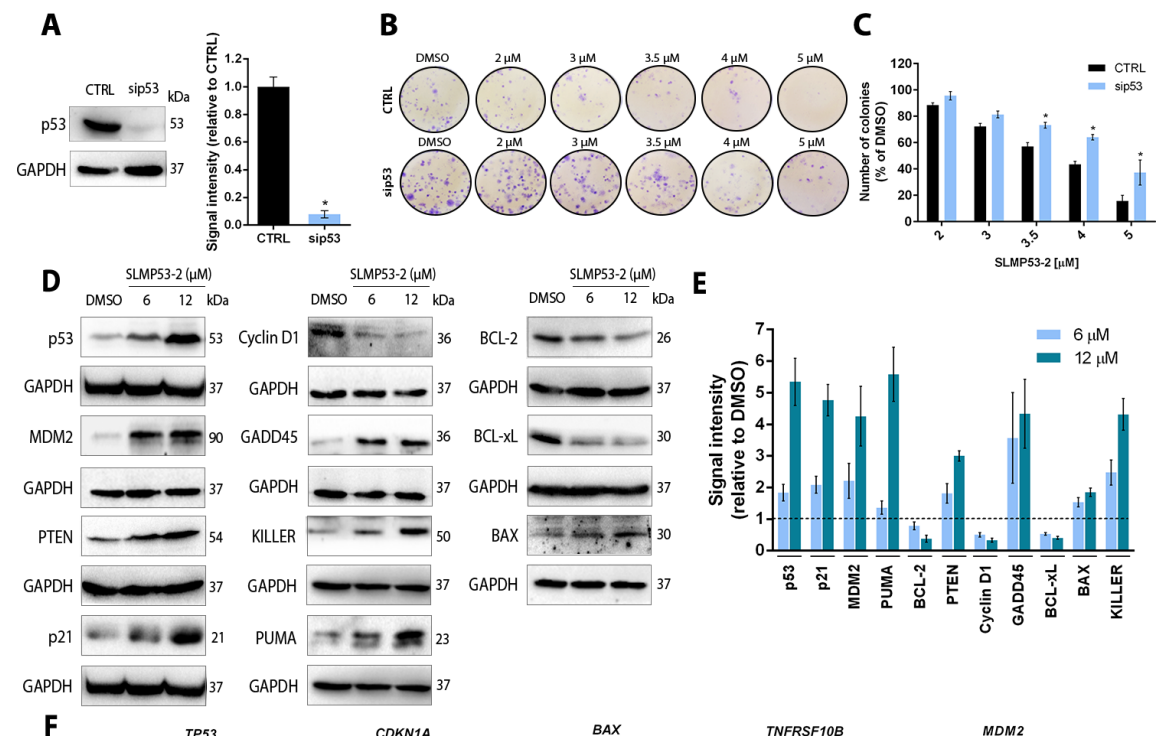

F

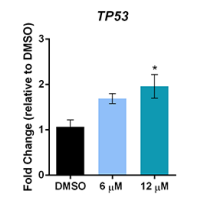

G
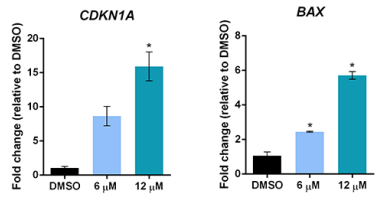

I

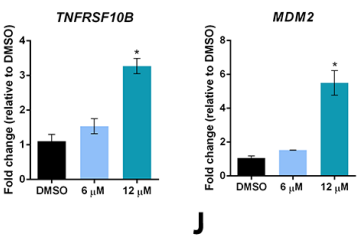

CHX (h)
SLMP53-2

\begin{tabular}{llll}
0 & 0.511 .52 \\
\hline & + & +
\end{tabular}

J

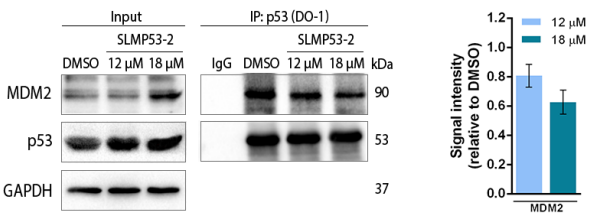

K
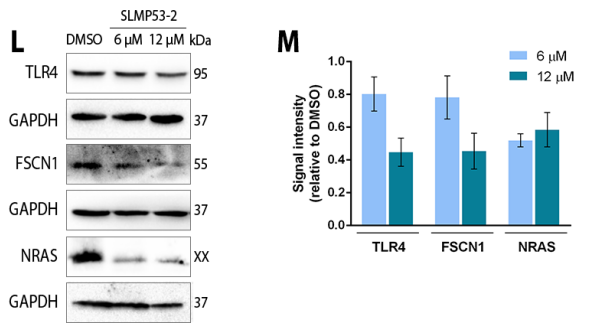
A

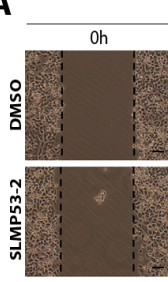

B

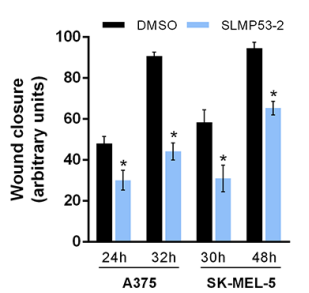

F

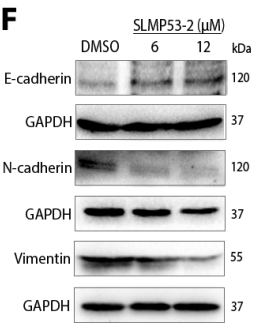

H

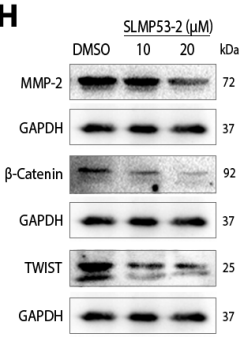

A

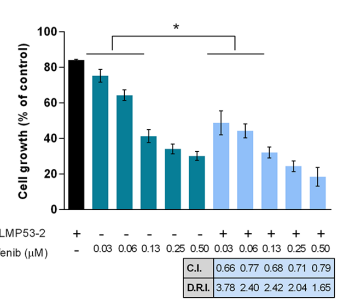

D

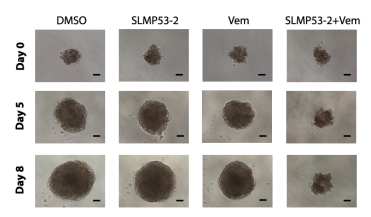

C
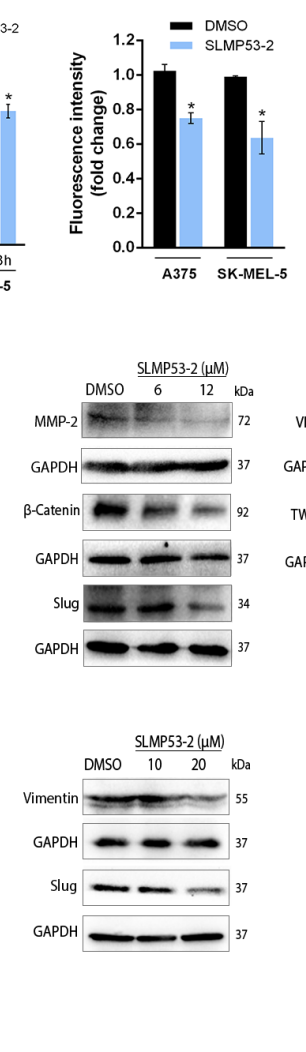

I

B

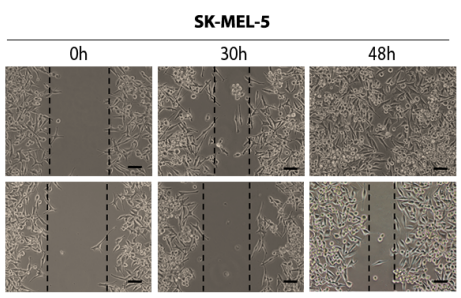

D

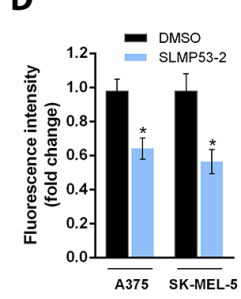

E $\quad$ 옹 - ำso

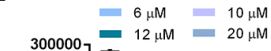

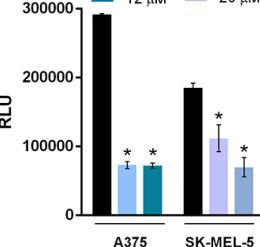

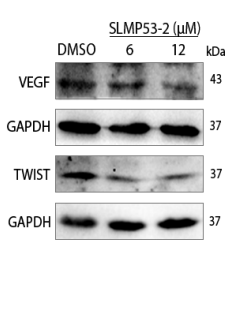
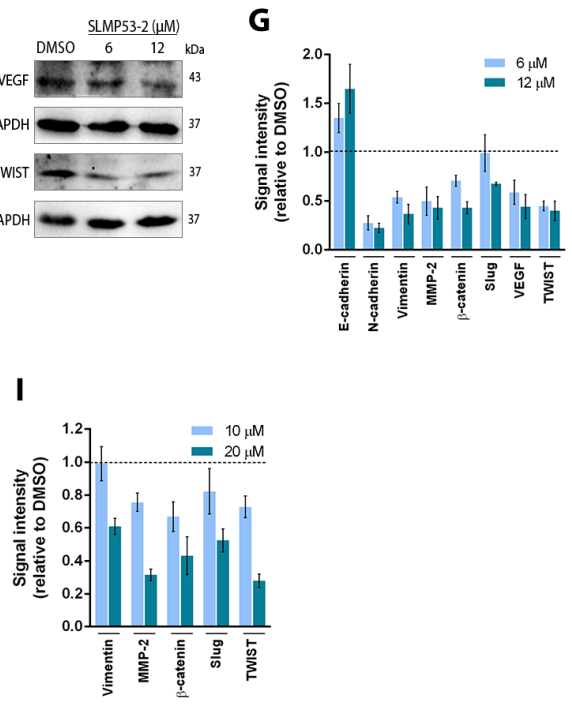

C
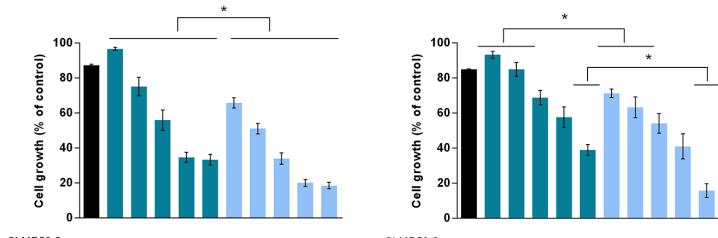

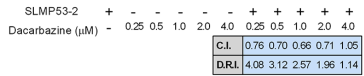

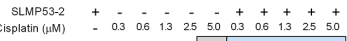

E

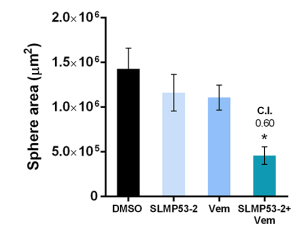


A

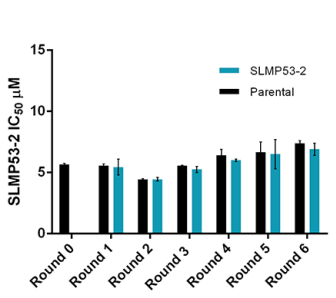

B

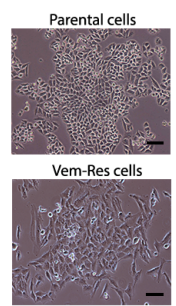

C

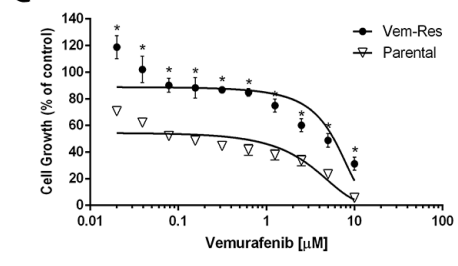

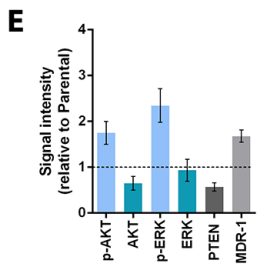

D Parental Vem-Res kDa

Parental Vem-Res $k D a$

ERK

$\longrightarrow 42$

GAPDH

$\longrightarrow 37$

AKT

GAPDH

Parental Vem-Res $k D a$

Parental Vem-Res $k D a$

2. 50 MDR-1 60

$\longrightarrow 37$ GAPDH $\longrightarrow$

$\longrightarrow 37$

G

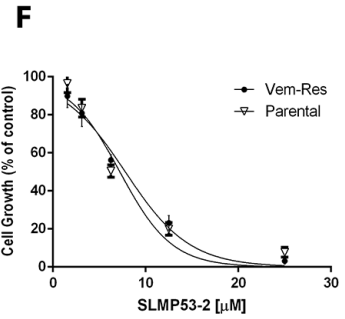

H
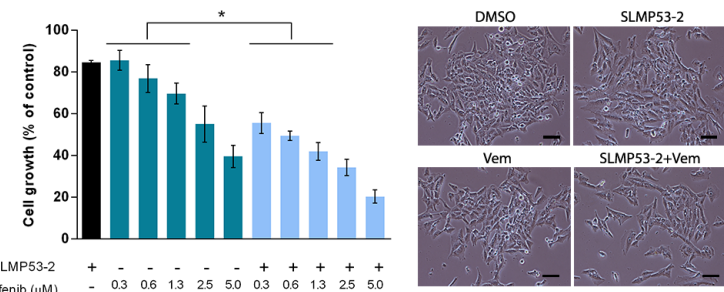

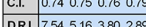

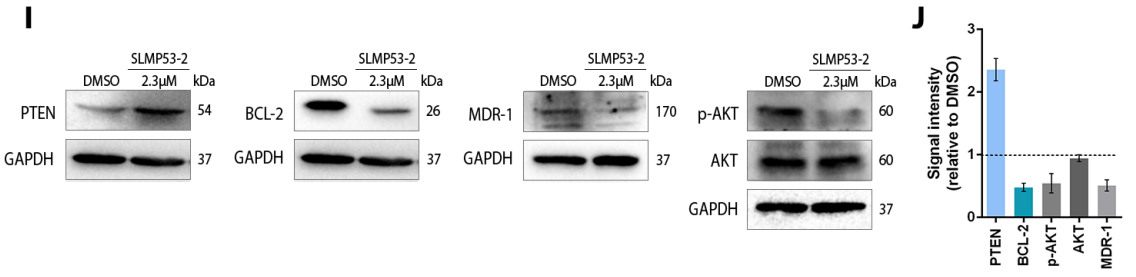


A
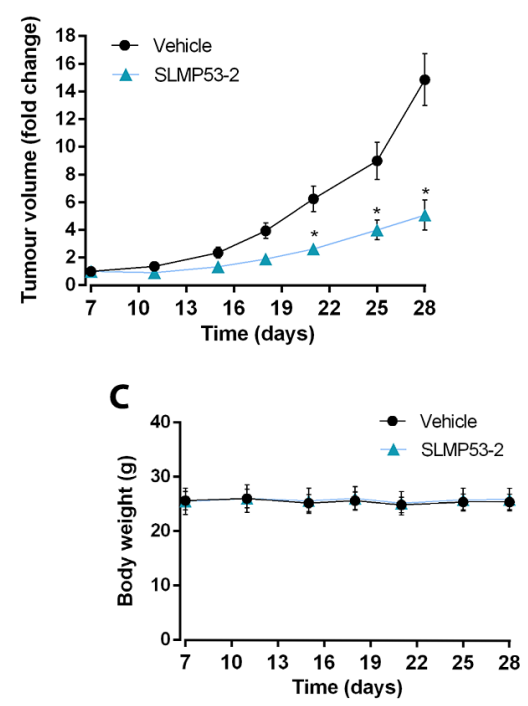

B
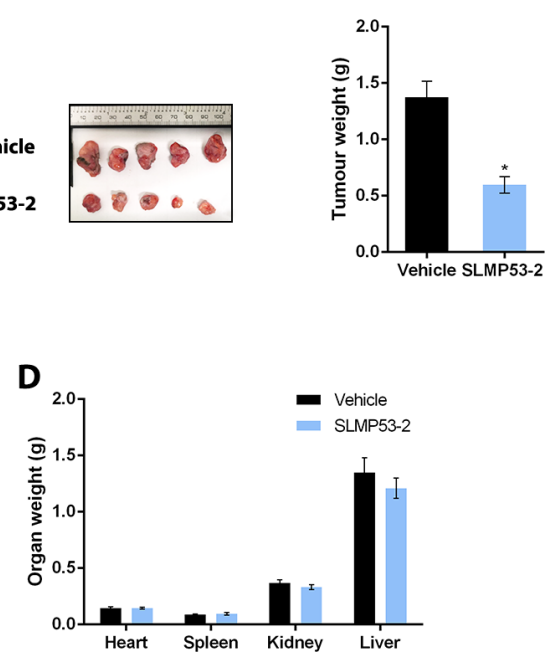

E
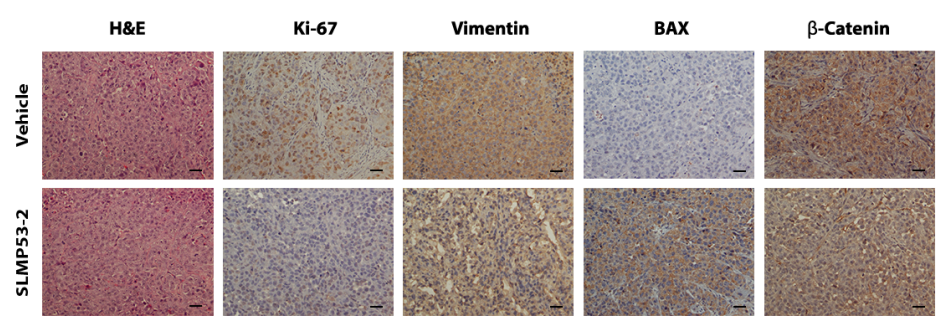

F
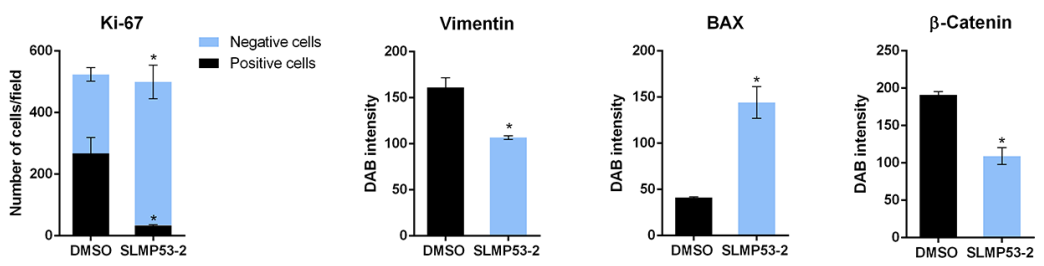